\title{
Asymptotic evolution of lipid vesicles
}

\author{
RICCARDO ROSSO ${ }^{\dagger}$ \\ Dipartimento di Matematica, Università di Pavia, via Ferrata 1, I-27100 Pavia, Italy
}

[Received 29 September 2000 and in revised form 11 December 2000]

\begin{abstract}
Dynamics of lipid vesicles is governed by an equation that offers many challenging mathematical problems, still not completely solved. In this paper we study vesicle dynamics by way of example. In a two-dimensional setting, when the vesicle is imagined as a closed curve, we draw a comparison with motion by curvature: it is shown that different decay laws govern the asymptotic relaxation to a circle. Finally, when the vesicle is modelled as an axisymmetric, compact surface, we study its relaxation to a sphere, to obtain the asymptotic shape and to illustrate the difficulties involved in dealing with this kind of evolution.
\end{abstract}

Keywords: Evolution of lipid vesicles; asymptotic vesicle dynamics; motion by curvature.

\section{Introduction}

Over the past three decades, the methods of mathematical physics have been employed more and more frequently to model many complex phenomena arising in biological systems. Among the numerous applications, modelling the behaviour of biological membranes, especially lipid membranes, clearly illustrates how these methods can lead to far-reaching results (see, for example, the rather extensive review by Seifert [25]). Lipid membranes live in an aqueous environment and are formed by amphiphilic molecules, also called amphiphiles, which owe their name to the ambiguous nature of their interaction with water. Indeed, they have an hydrophilic head, which is polar and can contain dipolar, ionic or zwitterionic groups, and an hydrophobic tail which consists of one or several alkyl chains (see Chapter 2 of Petrov [20]). Thus, when amphiphiles are put into a fluid environment, they tend to pack themselves forming bilayers, where the hydrophobic tails are masked by the hydrophilic heads, so that the contact with water is reduced. Sometimes, bilayers can bend forming closed membranes, called vesicles, where the contact between water and the hydrophobic portions of the amphiphiles is minimized. Besides vesicles, there are several other packings that are determined by many complex interactions: for a rather detailed description, see Chapter 17 of Israelachvili's book [13], for instance.

Here, we shall limit ourselves to consider vesicles, modelling them as compact surfaces in the ordinary three-dimensional Euclidean space. This is a reasonable approximation, because the thickness of the bilayer lies in the nanometre range, whereas the lateral dimensions of the vesicle are in the micrometre range. Since the molecules that form the vesicle have no inclination to leave it, the latter has a fixed area or, in other words, it is inextensible. It is also common in the literature to consider the volume enclosed by the vesicle as fixed. This attitude is justified when the time scale one is interested in is sufficiently small, as in the case of the thermal fluctuations of quasi-spherical vesicles, but it is possible to consider the vesicle as permeable, if the time scale is longer. In this

†Also at: Istituto Nazionale di Fisica della Materia, Sezione di Pavia. Email: Rosso@ dimat.unipv.it 
paper we will relax the constraint on the volume enclosed by a vesicle. The elastic properties of lipid vesicles have been described variously starting from the basic model introduced by Canham [2], which makes use of the following elastic free-energy functional:

$$
\mathcal{F}_{e l}[\mathcal{S}]:=\frac{\kappa}{2} \int_{\mathcal{S}} H^{2} \mathrm{~d} A,
$$

where $\mathcal{S}$ is the surface which represents the vesicle, $H$ its total curvature, $\mathrm{d} A$ the area measure, while $\kappa$ is a constitutive elastic modulus, usually referred to as the bending rigidity. By determining the critical points of $\mathcal{F}_{\text {el }}$, subject to the geometric constraint expressing inexstensibility, it is possible to find the equilibrium configurations taken by vesicles. Recently, more refined models have been proposed which take into account at different levels of accuracy the fact that the surface constituting the vesicle is not simply a flexible sheet, but is endowed with the bilayer structure. Nevertheless, the simple model based on (1.1) is able to capture the salient aspects of vesicle behaviour. The statics of vesicles has been explored thoroughly, and several rich catalogues of equilibrium shapes have been produced, according to the different models employed to describe the elastic response of vesicles: for some idea of the different approaches and results, see $[5,14,27]$. These catalogues have been mainly obtained under the assumption that the equilibrium shape is axisymmetric, to render the problem more tractable, and are based on numerical solutions of the equilibrium equation, since only a few particular explicit solutions are known: some results in this direction can be found in two papers by Ou-Yang $[17,18]$. An equilibrium equation which does not rely on the symmetry properties of the vesicles was obtained by Ou-Yang \& Helfrich [19], and has been generalized by Rosso \& Virga [23], to encompass more general free-energy densities.

The interest in vesicle dynamics also arose long ago, since the paper by Schneider et al. [24] concerning dynamical fluctuations of quasi-spherical vesicles made its appearance. In that paper, as well as in its generalization by Milner \& Safran [16], the force acting on the vesicle was used to write the appropriate boundary conditions for the Stokes equation at the vesicle surface, which is viewed as an interface separating two adiacent fluids. Since the dynamics of vesicles are overdamped, and so inertial effects can be neglected, it is crucial to single out the various dissipative mechanisms, establishing a hierarchy among them. In this perspective, Foltin [9] and Dörries \& Foltin [8] studied dynamics when the relevant dissipation is due to the internal friction between the layers forming the vesicle, while Cai \& Lubensky [1] considered the case where dissipation is due only to the interaction with the ambient fluid. A third mechanism arises from permeation through the membrane, but it seems to play a minor role (see [26]). When the size of the vesicles is in the micrometre range, which is the case considered here, hydrodynamic dissipation is the prevailing mechanism.

The dynamics of vesicles offers many formidable problems, because of the interactions with the ambient fluid. No explicit solutions of the evolution equation for impermeable vesicles derived by Seifert [26] are known, and analytical conclusions can be drawn only within particular approximations. For instance, in [26] Seifert considers the coupling with the fluid motion, obtaining a formal solution of the Stokes equation, which is then applied to study the deformations of a quasispherical vesicle in the presence of a shear flow, by use of the Lamb approximation, through an approach similar to that followed in [24]. A different avenue was followed by Rosso et al. [22], who examined the evolution of vesicles in the presence of an adhesive potential. They derived the evolution equations starting from a suitable dissipation principle, under the hypothesis that the fluid environment acts uniquely as a source of dissipation. This approach is reminiscent of the Rouse model adopted in polymer dynamics (see Section 4.1 of [7]) and introduced in the context of vesicle 
motion by Cai \& Lubensky [1]. Though the analysis of Rosso et al. is focused essentially on vesicles in two dimensions, they also derived the evolution equations for vesicles in three dimensions, for general elastic free-energy density and adhesion potential.

This paper aims at drawing mathematicians' attention towards a new class of evolving interfaces. In particular, it illustrates by example the asymptotic dynamics of vesicles, also showing the differences between this and the motion by curvature. To this aim, the paper is organized as follows. Section 2 contains the main ideas underlying the evolution of vesicles. As remarked in [22], this evolution shares many features with motion by Laplacian of curvature, introduced in surface dissipation. On the other hand, deep differences exist with motion by curvature, as shown by the asymptotic relaxation to a circle of a curve in two dimensions with normalized length. The decay law in this is quite different from that obtained in [22] for two-dimensional vesicles. Furthermore, in Sections 3 and 4 we study analytically the relaxation of a vesicle to a sphere, under the simplifying assumption that during the evolution the vesicle's shape stays axisymmetric. In this way, it is possible to determine explicitly the relaxation times associated with the normal modes, avoiding the need to compute the complete evolution. The mode corresponding to the longest relaxation time yields the asymptotic shape through which the vesicle approaches the spherical equilibrium solution. A similar, but much simpler, analysis was performed in [22] for the two-dimensional case, and the results were used to support the outcomes of numerical simulations based on the complete equations. In Section 3, the evolution equation is linearized by resort to a suitable parametrization of axisymmetric vesicles near a sphere; then, in Section 4, we retrace the normal modes along with their relaxation times, so that the asymptotic decay to a sphere can be studied in detail. Moreover, similarities and differences with the two-dimensional case are briefly discussed. Although confined to an ideal situation, this study sheds some light on the largely unexplored realm of vesicle dynamics and also offers some guidance to numerical results relying on the complete evolution equation. In Section 5 , we summarize the results of the paper.

\section{Vesicle dynamics against motion by curvature}

In this section, we collect some result on the evolution equation for vesicles both in two and three space dimensions. In the two-dimensional case we will stress some differences with motion by curvature, both qualitatively and quantitatively. We first summarise the main ideas underlying the study of vesicle dynamics, according to the approach followed by Rosso et al. [22]. To determine the evolution equation, a dissipation principle has been used, which posits that the mechanical energy stored in a control region containing the vesicle varies only because of dissipative effects. After examining different contributions to the mechanical energy and to the dissipation, and a scrutiny of their mutual relevance, it was possible to reduce the mechanical energy of the vesicle to its elastic energy and the dissipation to that due to the interaction with the ambient fluid. This fluid is imagined as motionless and not perturbed by the vesicle motion. On the other hand, it deforms the vesicle and dissipates part of its energy. In the simple case when the vesicle does not interact with any adhesive wall, and (1.1) is used to model the elastic energy, the evolution equation reduces to

$$
\kappa \Delta_{\mathrm{s}} H-H\left(\alpha_{0}-\frac{\kappa}{2} H^{2}\right)-2 \kappa K H=\gamma_{\nu} v_{\nu} .
$$

Here, $H:=\sigma_{1}+\sigma_{2}$ and $K:=\sigma_{1} \sigma_{2}$ are, respectively, the total and the Gaussian curvature of the vesicle, expressed in terms of its principal curvatures $\sigma_{1}$ and $\sigma_{2}$. The differential operator $\Delta_{\mathrm{s}}$ is the surface Laplacian, or Laplace-Beltrami operator on the vesicle (see, for example, p. 84 of [15]), 
whereas the constant $\alpha_{0}$ is a Lagrange multiplier which accounts for the inextensibility constraint. Finally, the coefficient $\gamma_{v}$ is a phenomenological friction coefficient, which accounts for the energy dissipated in the motion along the normal $\boldsymbol{\nu}$ to the vesicle surface. We note that although (2.1) is valid when the vesicle is permeable, adding a further multiplier $\mu(t)$ on the left-hand side of (2.1) would make it suitable also for impermeable vesicles, as shown in [22]. In the two-dimensional case, the vesicle is represented by a closed curve $c$ whose length is fixed, and equation (2.1) becomes

$$
\kappa \sigma^{\prime \prime}+\sigma\left(\frac{\kappa}{2} \sigma^{2}-\alpha_{0}\right)=-\gamma_{\nu} v_{\nu},
$$

where $\sigma$ is the curvature of $c$ and a prime has been used to denote differentiation with respect to the arc length $s$ along $c$. Since equations (2.1) and (2.2) depend on the second derivative of the curvature, they resembles the motion by Laplacian of curvature, which has been studied in many contexts (for an account, oriented to numerical applications, the reader is referred to [3]):

$$
\sigma^{\prime \prime}=v_{n} .
$$

At variance with motion by curvature, equations (2.2) and (2.3) do not preserve convexity of a curve, as was proved by means of numerical simulations in [22] and [4], respectively.

The equation governing motion by curvature can be written as

$$
\frac{\partial C}{\partial t}=\beta \sigma \nu,
$$

where $\nu$ is the normal unit vector to the curve $C$ and $\beta$ is a constant factor whose dimensions are $[\beta]=\left[L^{2} T^{-1}\right]$. This factor has been introduced to avoid using time scaling which is dimensionally uncorrect. A theorem by Grayson [12] states that a solution of (2.4) exists up to a critical time $T$ and that, as $t \rightarrow T$, it collapses to a point, with the limiting shape of a round circle. Although motion by curvature does not preserve the length of curves, a comparison with (2.2) is still possible, by adapting an argument of Gage [10]. Indeed, he noted that if the one-parameter family of curves evolving according to motion by curvature is normalized by homothetic expansion in the plane, so that each curve encloses area $\pi$, then the resulting family will converge to a circle (see p. 357 of [10]). We can follow the same line of thought and use a time-dependent scaling that renormalizes the length of the curve to a fixed value $L$. To determine the correct choice of the scaling factor $\lambda(t)$, we need to know some details about the evolution in time of $L_{0}(t)$, the length of a curve $C_{t}$ according to (2.4). It has been proved by Gage that $L_{0}(t)$ evolves according to

$$
\dot{L}_{0}(t)=-\beta \int_{C_{t}} \sigma^{2} \mathrm{~d} s,
$$

where a dot stands for time differentiation. As a consequence, the scaling factor $\lambda(t)$ must satisfy

$$
\lambda(t) L=L_{0}(t),
$$

which, by use of (2.5), gives

$$
\lambda(t)=\frac{\beta}{L} \int_{t}^{T} \mathrm{~d} \tau \int_{C_{\tau}} \sigma^{2} \mathrm{~d} s,
$$


where $T$ is the time needed to $C_{t}$ to collapse to a point. The curvature of a curve evolving according to (2.4) obeys the equation [11]

$$
\frac{\partial \sigma}{\partial t}=\beta\left(\frac{\partial^{2} \sigma}{\partial s^{2}}+\sigma^{3}\right) .
$$

The rescaling law (2.6) affects both the arc length $s$ and the curvature $\sigma$, according to

$$
\left\{\begin{array}{l}
\sigma \mapsto \bar{\sigma}:=\lambda \sigma \\
s \mapsto \bar{s}:=\frac{s}{\lambda}
\end{array}\right.
$$

so that by use of (2.8) we arrive at

$$
\frac{\partial \bar{\sigma}}{\partial t}=\frac{1}{\lambda} \dot{\lambda} \bar{\sigma}+\frac{\beta}{\lambda^{2}}\left(\frac{\partial^{2} \bar{\sigma}}{\partial \bar{s}^{2}}+\bar{\sigma}^{3}\right) .
$$

Let us consider a curve whose scaled curvature $\bar{\sigma}$ does not differ much from that of a circle with length $L=2 \pi R$ :

$$
\bar{\sigma}(\bar{s}, t)=\frac{1}{R}+\varepsilon u(\bar{s}, t) .
$$

Direct computations based on (2.6), (2.7) and (2.11), yield

$$
\lambda(t)=\frac{\beta}{R^{2}} \int_{t}^{T} \frac{1}{\lambda(\xi)} \mathrm{d} \xi
$$

or

$$
\frac{\mathrm{d}}{\mathrm{d} t} \lambda^{2}(t)=-\frac{2 \beta}{R^{2}},
$$

up to second-order terms in $\varepsilon$. Here, we have noted that the closure of the curve requires $\int_{0}^{L} u(\bar{s}, t) \mathrm{d} \bar{s}=0$. Thus, imposing $\lambda(T)=0$ we obtain

$$
\lambda^{2} R^{2}=2 \beta(T-t)
$$

which, once inserted in (2.10), yields

$$
2(T-t) \frac{\partial \bar{\sigma}}{\partial t}=-\bar{\sigma}+R^{2}\left(\frac{\partial^{2} \bar{\sigma}}{\partial \bar{s}^{2}}+\bar{\sigma}^{3}\right) .
$$

It is useful to introduce a dimensionless time $\tau$ such that

$$
\frac{\mathrm{d} \tau}{\mathrm{d} t}:=\frac{1}{T-t}, \quad \text { for } t<T .
$$

In terms of this new variable, inserting (2.11) into (2.10) and retaining only first-order terms in $\varepsilon$, we arrive at the linearized equation

$$
2 \frac{\partial u}{\partial \tau}=-u+R^{2} \frac{\partial^{2} u}{\partial \bar{s}^{2}} .
$$



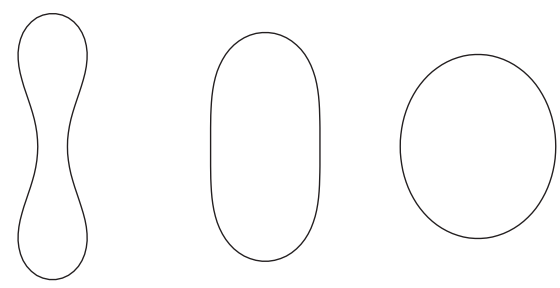

FIG. 1. Slowest mode in the decay of a two-dimensional vesicle to a circle.

Since we are only interested in determining the relaxation times, we consider the following ansatz:

$$
u_{n}(\bar{s}, \tau)=T_{n}(\tau) v_{n}(\bar{s}),
$$

with

$$
c_{n}(\bar{s}):=\alpha_{n} \sin \frac{n \bar{s}}{R}+\beta_{n} \cos \frac{n \bar{s}}{R} .
$$

Inserting (2.15) into (2.14), we immediately obtain an equation for $T(\tau)$

$$
\dot{T}_{n}(\tau)=-\frac{n^{2}+1}{2} T_{n}(\tau) .
$$

Geometrical considerations based on the four-vertex theorem - see Section 3 of [22]—lead us to conclude that the lowest value of $n$ which guarantees the closure of the curve is $n=2$. Thus, the asymptotic relaxation time is $\tau_{2}=\frac{5}{2}$. If we recall (2.13), and impose $\tau=0$ when $t=0$, we obtain

$$
\tau=-\ln \left(1-\frac{t}{T}\right)
$$

whence

$$
T_{2}(\tau) \mapsto T_{2}(t)=\left(1-\frac{t}{T}\right)^{-5 / 2} .
$$

This power-law decay is totally different from the exponential decay found in [22] on studying the analogous problem for (2.2). This is a neat feature that distinguishes evolution of vesicles from motion by curvature. Figure 1 shows three snapshots of the slowest mode in the decay of a vesicle in two space dimensions, for increasing values of time: they clearly exhibit the evolution towards a circular limit.

\section{Asymptotic vesicle dynamics}

Here, turning our attention back to a three-dimensional setting, we aim at illustrating the asymptotic dynamics of a vesicle that is a slight perturbation of a sphere. Since the area has a fixed value $A$, we take $A=4 \pi R^{2}, R$ being the radius of the asymptotic sphere. We assume that the vesicle stays axisymmetric during the whole evolution. We begin by linearizing equation (2.1). The constant $\alpha_{0}$ is determined by requiring the normal component $v_{v}$ of the velocity field to satisfy

$$
\int_{\mathcal{S}} H v_{\nu} \mathrm{d} a=0,
$$



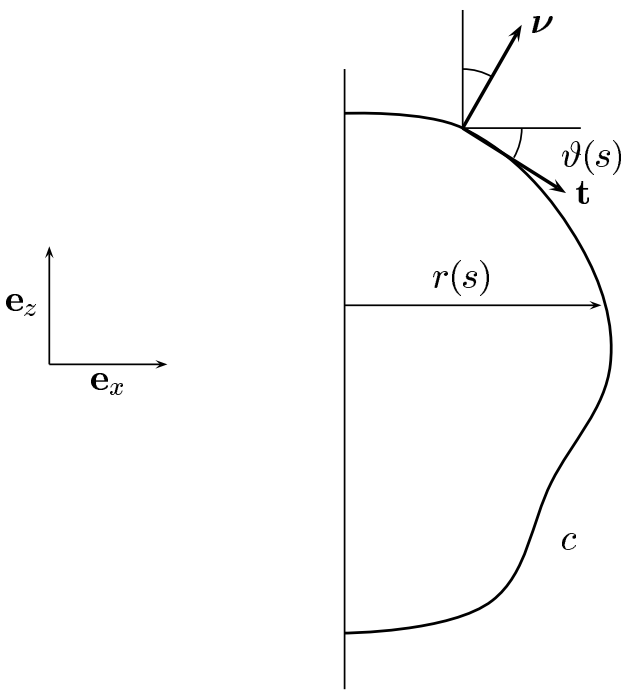

FIG. 2. The contour $c$ generating an axisymmetric vesicle.

which is a necessary condition for $A$ to stay constant. Indeed, if we decompose the velocity field $\mathbf{v}$ as $\mathbf{v}=\mathbf{v}_{s}+v_{\nu} \boldsymbol{\nu}$, where $\mathbf{v}_{S}$ denotes the tangential component of $\mathbf{v}$, inextensibility of the vesicle requires

$$
\operatorname{div}_{s} \mathbf{v}=\operatorname{div}_{s} \mathbf{v}_{s}+H v_{v}=0
$$

and so, by integration on $\mathcal{S}$ and use of the surface-divergence theorem (see for example, p. 87 of [28]), we obtain condition (3.1). For completeness, we recall that the surface gradient of a scalar field $g$ defined on $\mathcal{S}$ is given by

$$
\nabla_{s} g:=\mathbf{P} \nabla g,
$$

where $\mathbf{P}:=\mathbf{I}-\boldsymbol{\nu} \otimes \boldsymbol{\nu}$ is the orthogonal projector on the local tangent plane to $\mathcal{S}$. Similarly, the surface gradient of a vector field $\mathbf{u}$ on $\mathcal{S}$ is defined as

$$
\nabla_{s} \mathbf{u}:=\nabla \mathbf{u P} .
$$

Finally, the surface divergence of a vector field is simply the trace of its surface gradient. Thus, we can write $\Delta_{s} g=\operatorname{div}_{s} \nabla_{s} g$.

Since we are concerned with evolution in an axisymmetric class, we only need to know the motion of the contour $c$ that generates the vesicle (see Figure 2).

Let $s$ be the arc length along $c$ and let $r(s, t)$ be the distance from the symmetry axis of a point lying on the contour. Since we are perturbing a sphere, we can set

$$
r(s, t)=R \sin \frac{s}{R}+\varepsilon f(s, t),
$$

where $\varepsilon$ is a small parameter, and $f(s, t)$ an unknown function. The angle $\vartheta$ between the unit tangent $\mathbf{t}$ to $c$ and $\mathbf{e}_{x}$ is related to $r$ via the relation

$$
\cos \vartheta(s, t)=r_{, s}=\cos \frac{s}{R}+\varepsilon f_{, s},
$$


where a comma denotes partial differentiation. To find the function $\vartheta(s, t)$, we define

$$
\vartheta(s, t)=: \frac{s}{R}+\varepsilon u(s, t),
$$

for some function $u$. Evaluating $\cos \vartheta$ by means of (3.5) and comparing the result with (3.4), we obtain

$$
u(s, t)=-\frac{f_{, s}}{\sin \frac{s}{R}} .
$$

The apparent singularity at $s=0$ is ruled out by requiring that $f_{, s}(0, t)$ vanishes at least as $s$, near the pole $s=0$. We note in passing that $f(0, t)=0$, for consistency with (3.3). On the other hand, we stress that, although the area of the vesicle is not allowed to change, the length of the contour $c$ does not have a prescribed value. In other words, on the perturbed shape $s$ ranges in an interval $\left[0, s_{M}\right]$, where the upper limit $s_{M}$ is unknown, and has to be found by properly fixing the value of the area. Later on, we shall see how to determine $u_{M}:=u\left(s_{M}, t\right)$ and how to circumvent the apparent singularity in (3.6) when $s=\pi$, by resorting to the analytic solution of the linearized problem.

It is well known that the meridians and the parallels of an axisymmetric surface are its lines of curvature. Standard computations based on (3.3) and (3.4) yield the corresponding values of the principal curvatures $\sigma_{1}$ and $\sigma_{2}$,

$$
\begin{aligned}
& \sigma_{1}:=\vartheta_{, s}=\frac{1}{R}-\varepsilon \frac{f_{, s s} \sin \frac{s}{R}-\frac{f_{s s}}{R} \cos \frac{s}{R}}{\sin ^{2} \frac{s}{R}}+\mathrm{o}(\varepsilon) \\
& \sigma_{2}:=\frac{\sin \vartheta}{r}=\frac{1}{R}-\varepsilon\left[\frac{f}{R^{2} \sin \frac{s}{R}}+\frac{f_{, s} \cos \frac{s}{R}}{R \sin ^{2} \frac{s}{R}}\right]+\mathrm{o}(\varepsilon),
\end{aligned}
$$

whence we readily obtain the expression of the total and the Gaussian curvature of the vesicle:

$$
\begin{aligned}
& H:=\sigma_{1}+\sigma_{2}=\frac{2}{R}-\varepsilon\left[\frac{f_{, s s}}{\sin \frac{s}{R}}+\frac{f}{R^{2} \sin \frac{s}{R}}\right]+\mathrm{o}(\varepsilon) \\
& K:=\sigma_{1} \sigma_{2}=\frac{1}{R^{2}}-\varepsilon\left[\frac{f}{R^{3} \sin \frac{s}{R}}+\frac{f_{, s s}}{R \sin \frac{s}{R}}\right]+\mathrm{o}(\varepsilon) .
\end{aligned}
$$

To proceed further, we need the expression of the surface Laplacian $\Delta_{S}$ on the vesicle. By its definition, and exploiting the axisymmetry of the vesicle we obtain

$$
\Delta_{s}:=\partial_{s s}^{2}+\frac{\cos \vartheta}{r} \partial_{s}+\frac{1}{r^{2}} \partial_{\varphi \varphi}^{2}
$$

where $\varphi$ is the azimuthal angle. Since in (2.1) $\Delta_{s}$ acts only on $H$, which is independent of $\varphi$, we can ignore the last term in the expression of the surface Laplacian and so, by use of (3.3) and (3.4), here we write

$$
\Delta_{s}=\partial_{s s}^{2}+\frac{1}{R} \cot \frac{s}{R} \partial_{s}+\varepsilon\left[\frac{f_{, s}}{R \sin \frac{s}{R}}-\frac{f \cos \frac{s}{R}}{R^{2} \sin ^{2} \frac{s}{R}}\right] \partial_{s}+\mathrm{o}(\varepsilon) .
$$

Since the zeroth order of $H$ is constant we have, up to first-order terms in $\varepsilon$

$$
\Delta_{s} H=-\varepsilon\left(\partial_{s s}^{2}+\cot \frac{\vartheta}{R} \partial_{s}\right)\left(\frac{f_{, s s}}{\sin \frac{s}{R}}+\frac{f}{R^{2} \sin \frac{s}{R}}\right) .
$$


It is easy to show from equations (3.8) that $\frac{1}{2} H^{3}-2 K H=\mathrm{o}(\varepsilon)$, so that the linearized equation (2.1) becomes

$$
-H \alpha_{0}+\Delta_{s} H=-\frac{\gamma_{\nu}}{\kappa} v_{\nu} .
$$

To compute $\alpha_{0}$, we multiply (3.10) by $H$ and integrate on the vesicle surface $\mathcal{S}$ : by recalling (3.1), we easily obtain

$$
\int_{\mathcal{S}} H \Delta_{S} H \mathrm{~d} a=\alpha_{0} \int_{\mathcal{S}} H^{2} \mathrm{~d} a
$$

Moreover, by the identity

$$
H \Delta_{s} H=\operatorname{div}_{s}\left(H \nabla_{s} H\right)-\left(\nabla_{s} H\right)^{2},
$$

and since $\mathcal{S}$ has no border and $\nabla_{s} H$ is a tangential vector field, by the surface-divergence theorem we conclude that

$$
\int_{\mathcal{S}} \operatorname{div}_{S}\left(H \nabla_{S} H\right) \mathrm{d} a=0 .
$$

Thus, since $H^{2}$ has a non-vanishing zeroth-order term, whereas $\nabla_{S} H$ is first order in $\varepsilon, \alpha_{0}$ must have a vanishing zeroth-order term: this conclusion can be obtained through a simple inspection of (2.1), by requiring a sphere to be an equilibrium solution. Similarly, since (3.11) now reads as

$$
-\int_{\mathcal{S}}\left(\nabla_{s} H\right)^{2} \mathrm{~d} a=\alpha_{0} \int_{\mathcal{S}} H^{2} \mathrm{~d} a,
$$

and the left-hand side is $\mathrm{O}\left(\varepsilon^{2}\right)$, also the first-order correction to $\alpha_{0}$ must vanish. Thus, we can recast (3.10) as

$$
\Delta_{s} H_{1}=-\frac{\gamma_{v}}{\kappa} v_{v},
$$

$H_{1}$ being the first-order correction to the total curvature $H$.

For later use, we now discuss the condition that $f(s, t)$ must satisfy to obey the constraint on the area of $\mathcal{S}$. By Pappus' theorem (see, for example, p. 101 of [6]), we can write this constraint as

$$
4 \pi R^{2}=2 \pi \int_{0}^{s_{M}} r(s) \mathrm{d} s,
$$

where, by use of (3.5), we can set $s_{M}=\pi R-\varepsilon u_{M}$. By computing the integral in (3.13) with the aid of (3.3), we have

$$
-\left.2 \pi R^{2}\left[\cos \frac{s}{R}\right]\right|_{0} ^{\pi R-\varepsilon u_{M}}+2 \pi \varepsilon \int_{0}^{\pi R} f(s, t) \mathrm{d} s+\mathrm{o}(\varepsilon)=0 .
$$

Thus, up to first-order terms in $\varepsilon$, the perturbed vesicle has the same area as the sphere, provided that

$$
\int_{0}^{\pi R} f(s, t) \mathrm{d} s=0
$$


To solve (3.12), we need another equation relating $v_{v}$ and $H$, and this can be done by determining the evolution of $H$ itself. As shown in [22],

$$
\frac{\partial H}{\partial t}=\left(\nabla_{s} \boldsymbol{\nu} \cdot \nabla_{s} \mathbf{v}+\operatorname{div}_{s} \mathbf{a}\right)
$$

where the vector field $\mathbf{a}$ is defined as $\mathbf{a}:=\left(\nabla_{s} \mathbf{v}\right)^{\mathrm{T}} \boldsymbol{\nu}$ and the superscript ${ }^{\mathrm{T}}$ denotes the transposition operator. In the next section, we combine (3.15) with (3.12) to obtain a single equation involving only $H_{1}$.

\section{Normal modes}

The asymptotic dynamics of the vesicle is here resolved in its normal modes. To this aim, it suffices to consider a reduced velocity field with no azimuthal component. This assumption, which guarantees that the evolution preserves the axisymmetry, can be slightly relaxed, as shown later in this section. For the time being, we restrict attention to velocity fields such that

$$
\mathbf{v}=v_{t} \mathbf{t}+v_{\nu} \boldsymbol{\nu}
$$

Direct computations show that

$$
\nabla_{s} \mathbf{v}=v_{t} \nabla_{s} \mathbf{t}+\mathbf{t} \otimes \nabla_{s} v_{t}+v_{\nu} \nabla_{s} \boldsymbol{\nu}+\boldsymbol{\nu} \otimes \nabla_{s} v_{\nu}
$$

Here, the surface gradient $\nabla_{S} \nu$ of the outer normal to $\mathcal{S}$ is expressed as (see, for example, p. 87 of [28])

$$
\nabla_{s} \boldsymbol{\nu}=\sigma_{1} \mathbf{t} \otimes \mathbf{t}+\sigma_{2} \mathbf{e}_{\varphi} \otimes \mathbf{e}_{\varphi}
$$

where $\mathbf{e}_{\varphi}$ is the unit tangent vector to the local parallel on $\mathcal{S}$, oriented so that $\mathbf{e}_{\varphi} \wedge \mathbf{t}=\boldsymbol{\nu}$. By the definition of total and Gaussian curvature we readily obtain that

$$
\nabla_{s} \mathbf{v} \cdot \nabla_{s} \boldsymbol{\nu}=v_{v}\left(H^{2}-2 K\right)+v_{t} \nabla_{s} \mathbf{t} \cdot \nabla_{s} \boldsymbol{\nu}+\sigma_{1} \mathbf{t} \cdot \nabla_{s} v_{t}
$$

To compute the remaining terms in (3.15) and (4.1), it is expedient to introduce the Darboux trihedron $\left\{\mathbf{t}, \boldsymbol{\nu}, \boldsymbol{\nu}_{s}\right\}$ along $c$, (see, for example, p. 261 of [6]) with $\boldsymbol{\nu}_{s}:=\boldsymbol{\nu} \wedge \mathbf{t}=-\mathbf{e}_{\varphi}$. Darboux formulae can be used to express the rate of variation along $c$ of the vectors forming the Darboux trihedron. In the general case they can be written as

$$
\left\{\begin{array}{l}
\frac{\mathrm{d} \mathbf{t}}{\mathrm{d} s}=\kappa_{g} \boldsymbol{\nu}_{s}+\kappa_{n} \boldsymbol{\nu} \\
\frac{\mathrm{d} \nu_{s}}{\mathrm{~d} s}=-\kappa_{g} \mathbf{t}-\tau_{g} \boldsymbol{\nu} \\
\frac{\mathrm{d} \nu}{\mathrm{d} s}=-\kappa_{n} \mathbf{t}+\tau_{g} \boldsymbol{\nu}_{s}
\end{array}\right.
$$

where $\kappa_{n}$ and $\kappa_{g}$ are, respectively, the normal and the geodesic curvature of $c$, whereas $\tau_{g}$ is its geodesic torsion. In this case, the meridians of axisymmetric surfaces are both geodesics and lines of curvature, and so along them $\kappa_{g}=\tau_{g} \equiv 0$, while the normal curvature is just $-\sigma_{1}$. Hence, 
equations (4.2) become

$$
\left\{\begin{array}{l}
\frac{\mathrm{d} \mathbf{t}}{\mathrm{d} s}=-\sigma_{1} \nu \\
\frac{\mathrm{de} \mathbf{e}_{\varphi}}{\mathrm{d} s}=\mathbf{0} \\
\frac{\mathrm{d} \nu}{\mathrm{d} s}=\sigma_{1} \mathrm{t} .
\end{array}\right.
$$

A Darboux trihedron can be defined also on a parallel as $\left\{\mathbf{e}_{\varphi}, \boldsymbol{\nu}, \boldsymbol{\nu}_{S}\right\}$, where now $\boldsymbol{\nu}_{s}:=\boldsymbol{\nu} \wedge \mathbf{e}_{\varphi}=$ t. In general, parallels are lines of curvature, but they are not geodesics, and so only the geodesic torsion vanishes identically along them. If we introduce the curvilinear abscissa $\bar{s}$ along a parallel, Darboux formulae along it read as

$$
\left\{\begin{array}{l}
\frac{\mathrm{d} \mathbf{e}_{\varphi}}{\mathrm{d} \bar{s}}=\kappa_{g} \mathbf{t}-\sigma_{2} \nu \\
\frac{\mathrm{d} \mathbf{t}}{\mathrm{d} \bar{s}}=-\kappa_{g} \mathbf{e}_{\varphi} \\
\frac{\mathrm{d} \nu}{\mathrm{d} \bar{s}}=\sigma_{2} \mathbf{e}_{\varphi} \cdot
\end{array}\right.
$$

Here, $\kappa_{g}$ is the geodesic curvature of the local parallel.

By definition of surface gradient, we can recast (4.3) and (4.4), respectively, as

$$
\left\{\begin{array}{l}
\left(\nabla_{s} \mathbf{t}\right) \mathbf{t}=-\sigma_{1} \nu \\
\left(\nabla_{s} \mathbf{e}_{\varphi}\right) \mathbf{t}=\mathbf{0} \\
\left(\nabla_{s} \boldsymbol{\nu}\right) \mathbf{t}=\sigma_{1} \mathbf{t},
\end{array}\right.
$$

and

$$
\left\{\begin{array}{l}
\left(\nabla_{s} \mathbf{e}_{\varphi}\right) \mathbf{e}_{\varphi}=\kappa_{g} \mathbf{t}-\sigma_{2} \boldsymbol{\nu} \\
\left(\nabla_{s} \mathbf{t}\right) \mathbf{e}_{\varphi}=-\kappa_{g} \mathbf{e}_{\varphi} \\
\left(\nabla_{s} \boldsymbol{\nu}\right) \mathbf{e}_{\varphi}=\sigma_{1} \mathbf{t} .
\end{array}\right.
$$

To obtain from these the expression for $\nabla_{s} \mathbf{t}$ we need only observe that $\left(\nabla_{s} \mathbf{t}\right)^{\mathrm{T}} \mathbf{t}=\mathbf{0}$, since $\mathbf{t} \cdot \mathbf{t}=1$, and that $\left(\nabla_{s} \mathbf{t}\right) \boldsymbol{\nu}=\mathbf{0}$. Hence, we obtain that

$$
\nabla_{s} \mathbf{t}=-\kappa_{g} \mathbf{e}_{\varphi} \otimes \mathbf{e}_{\varphi}-\sigma_{1} \boldsymbol{\nu} \otimes \mathbf{t}
$$

Similarly, since $\mathbf{e}_{\varphi} \cdot \mathbf{t}=0$ and $\mathbf{e}_{\varphi} \cdot \mathbf{e}_{\varphi}=1$, equations (4.5)2, (4.6) 1,2 , and (4.7) yield

$$
\left(\nabla_{s} \mathbf{e}_{\varphi}\right)=-\sigma_{2} \nu \otimes \mathbf{e}_{\varphi}+\kappa_{g} \mathbf{t} \otimes \mathbf{e}_{\varphi} .
$$

We are now in a position to recast (4.1) in the form

$$
\nabla_{s} \mathbf{v} \cdot \nabla_{s} \boldsymbol{\nu}=v_{v}\left(H^{2}-2 K\right)-\kappa_{g} \sigma_{2} v_{t}+\sigma_{1} \mathbf{t} \cdot \nabla_{s} v_{t}
$$

Moreover,

$$
\mathbf{a}=-\sigma_{1} v_{t} \mathbf{t}+\nabla_{s} v_{v}
$$


whence it follows that

$$
\operatorname{div}_{s} \mathbf{a}=\Delta_{s} v_{v}+\kappa_{g} \sigma_{1} v_{t}-\sigma_{1} \mathbf{t} \cdot \nabla_{s} v_{t}-\left(\mathbf{t} \cdot \nabla_{s} \sigma_{1}\right) v_{t}
$$

By inserting (4.9) and (4.10) into (3.15), we have

$$
\frac{\partial H}{\partial t}=\Delta_{s} v_{v}+v_{\nu}\left(H^{2}-2 K\right)+\kappa_{g}\left(\sigma_{1}-\sigma_{2}\right) v_{t}+\left(\mathbf{t} \cdot \nabla_{s} \sigma_{1}\right) v_{t},
$$

which can be further simplified if we keep only first-order terms in $\varepsilon$. Indeed, by (3.12) we obtain that $v_{v}$ is first-order in $\varepsilon$ and so, by use of (3.2), we also conclude that $\operatorname{div}_{s} v_{t}$ is first-order in $\varepsilon$. Thus, if we recall that both $\sigma_{1}-\sigma_{2}$ and $\nabla_{s} \sigma_{1}$ are first-order in $\varepsilon$, we obtain that

$$
\frac{\partial H_{1}}{\partial t}=\Delta_{s} v_{v}+\frac{2}{R^{2}} v_{\nu},
$$

where we have expanded $H^{2}-2 K$, keeping only the leading term. By substituting (3.12) into this equation, we finally arrive to an evolution equation for $H_{1}$ only:

$$
\frac{\partial H_{1}}{\partial t}=-\frac{\kappa}{\gamma_{\nu}}\left(\Delta_{s}^{2} H_{1}+\frac{2}{R^{2}} \Delta_{s} H_{1}\right)
$$

It is possible to rescale (4.12), by introducing the following dimensionless variables: $\eta:=\frac{s}{R}, h_{1}=$ $R H_{1}, \tau:=\frac{\kappa t}{\gamma_{\nu} R^{4}}$, and $\psi(s, \tau):=\frac{f(s, \tau)}{R}$. Thus, we obtain

$$
\frac{\partial h_{1}}{\partial \tau}=-\left(\Delta_{\eta}^{2} h_{1}+2 \Delta_{\eta} h_{1}\right)
$$

where $\Delta_{\eta}:=\frac{\partial^{2}}{\partial \eta^{2}}+\cot \eta \frac{\partial}{\partial \eta}$ is the scaled surface Laplacian on a sphere, deprived of the azimuthal component. It is now easy to compute the relaxation times by separating the variables: we set $h_{1}(\eta, \tau)=T(\tau) Y(\eta)$, with $Y(\eta)$ an eigenfunction of the operator on the right-hand side of (4.13), that is, any zonal spherical harmonic $Y_{\ell, 0}(\eta)$ defined as

$$
Y_{\ell, 0}(\eta)=P_{\ell}(\cos \eta), \ell \geqslant 0,
$$

where $P_{\ell}$ is the $\ell$ th Legendre polynomial. Recalling that

$$
\Delta_{\eta} Y_{\ell, 0}(\eta)=-\ell(\ell+1) Y_{\ell, 0}(\eta),
$$

we readily prove that $T(t)=\mathrm{e}^{-t / t_{\ell}}$, where we have reintroduced the original time variable $t$, and $t_{\ell}$ is given by

$$
t_{\ell}:=\frac{\gamma_{\nu} R^{4}}{\kappa(\ell-1) \ell(\ell+1)(\ell+2)}
$$

Apart from a different numerical prefactor, the relaxation times we have just found exhibit the same dependence on both the vesicle size $R$ and the constitutive parameters $\kappa$ and $\gamma_{v}$ as in the two-dimensional case. 
The normal modes corresponding to $\ell=0$ and $\ell=1$ look singular, since they would correspond to infinite relaxation times. However, these modes never occur, as they do not match the appropriate boundary conditions. By use of (3.8) 1 and proper scaling, for $\ell$ th mode we can write

$$
h_{1}(\eta)=a Y_{\ell, 0}(\eta)=-\frac{1}{\sin \eta}\left(\psi_{, \eta \eta}+\psi\right),
$$

where $a$ is an arbitrary constant. By setting for simplicity $a=-1$, the equation for the perturbation $\psi$ becomes

$$
\psi_{, \eta \eta}+\psi=Y_{\ell, 0}(\eta) \sin \eta .
$$

To determine $\psi$ we can apply the method of variation of parameters obtaining, by repeated integrations,

$$
\begin{aligned}
\psi(\eta)= & \alpha \sin \eta+\beta \cos \eta+\sin \eta \int_{0}^{\eta} \cos u \sin u Y_{\ell, 0}(u) \mathrm{d} u \\
& -\cos \eta \int_{0}^{\eta} \sin ^{2} u Y_{\ell, 0}(u) \mathrm{d} u,
\end{aligned}
$$

where the constants $\alpha$ and $\beta$ must be determined by imposing the boundary conditions $\psi(0)=0$, $\psi^{\prime}(0)=0$ : it is easy to see from (4.15) this is equivalent to enforce $\alpha=\beta=0$. If we integrate by parts (3.14) using (4.15), we obtain

$$
\begin{aligned}
& \int_{0}^{\pi} \psi(\eta) \mathrm{d} \eta=\int_{0}^{\pi} \sin \eta \cos \eta P_{\ell, 0}(\cos \eta) \mathrm{d} \eta \\
& +\int_{0}^{\pi} \sin \eta P_{\ell, 0}(\cos \eta) \mathrm{d} \eta=\frac{2}{3} \delta_{1 \ell}+2 \delta_{0 \ell},
\end{aligned}
$$

because of the othogonality properties of spherical harmonics. Thus, (3.14) can be satisfied if and only if $\ell \neq 0,1$.

To recover the asymptotic shape we need just to go back to (3.4), to obtain

$$
\varrho(\eta):=\frac{r(\eta)}{R}=\int_{0}^{\eta}\left(\cos u+\varepsilon \psi_{, u}\right) \mathrm{d} u,
$$

and

$$
\zeta(\eta):=\frac{z(\eta)}{R}=\int_{0}^{\eta}\left(\sin u-\varepsilon \psi_{, u} \cot u\right) \mathrm{d} u,
$$

where $z(\eta)$ is the distance of a point on $c$ from the rotation axis. In obtaining the last formula we have used the equation

$$
\sin \vartheta(s)=\sin \frac{s}{R}-\varepsilon f_{, s} \cot \frac{s}{R}+\mathrm{o}(\varepsilon),
$$

which readily follows from (3.5).

We finally compute the upper limit $\eta_{M}$ for $\eta$. We note that, when $\eta=\eta_{M}$, the angle $\vartheta$ reaches $\pi$ and $\varrho$ vanishes. Thus, it follows from (3.5) that

$$
\eta_{M}=\pi-\varepsilon u_{M} .
$$



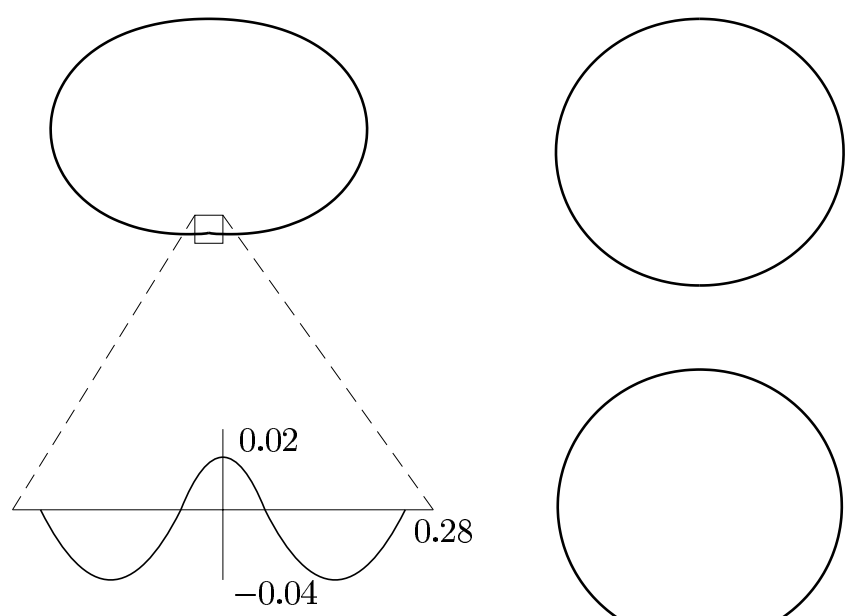

FIG. 3. Slowest mode in the decay to a sphere.

Moreover, the vanishing of $\varrho$ at the pole $\vartheta=\pi$ implies

$$
\sin \left(\pi-\varepsilon u_{M}\right)+\varepsilon \psi\left(\eta_{M}\right)=0
$$

whence, neglecting terms of order higher than one in $\varepsilon$, we get

$$
u_{M}=-\psi(\pi)
$$

By (4.15) and the symmetry properties of $Y_{\ell, 0}(u)$, it is easy to conclude that $\psi(\pi)=0$, when $\ell>2$ is odd, and so these modes do not change the length of $c$, at least to the first order. Finally, a straightforward computation and use of the orthogonality properties of spherical harmonics show that $\psi_{, \eta}$ goes to zero, as $\eta \rightarrow \pi$, whence the singularity in (3.6) at $s=\pi R$ is ruled out. When $\ell$ is even, it is possible to show (see formula 1 on p. 197 of Prudnikov et al. [21]) that

$$
\psi(\pi)=\int_{0}^{\pi} \sin ^{2} u Y_{\ell, 0}(u) \mathrm{d} u=2 \Gamma^{2}\left(\frac{3}{2}\right)_{3} F_{2}\left(\frac{3}{2},-\ell, \ell+1 ; 1,3,1\right)
$$

where $\Gamma$ is the Euler gamma function, and ${ }_{3} F_{2}$ is a hypergeometric function. In the simplest case when $\ell=2$ it is easy to compute $\psi(\pi)=-\frac{\pi}{16}$, whence $\eta_{M}=\pi\left(1-\frac{\varepsilon}{16}\right)$. Thus, for any $\varepsilon>0$, the troublesome value $\eta_{M}=\pi$ is never reached. Although the computations for higher even values of $\ell$ rapidly becomes involved, it is easy to show by use of (4.15), that $\psi_{, \eta}$ behaves like $\sin \eta$ when $\eta \rightarrow \pi$, so that no singularities arise in (3.6). The asymptotic shapes corresponding to $\ell=2$ are sketched in Figure 3, for increasing values of time.

The first image, in particular, suggests that the limiting shape has no equatorial symmetry, at variance with the two-dimensional case. A possible explanation could be the change in the geometric constraint on the profile $c$, which has no longer a fixed length.

To conclude this analysis, we have to prove that adding a non-zero component of the velocity along the azimuthal direction does not change the properties of the asymptotic evolution. Let us 
consider the velocity field $\mathbf{v}=v_{\varphi} \mathbf{e}_{\varphi}$. To preserve the axisymmetry of the vesicle, the function $v_{\varphi}$ must depend only on $s$, the arc length along the meridian. Then

$$
\nabla_{s} \mathbf{v}=v_{\varphi} \nabla_{s} \mathbf{e}_{\varphi}+\mathbf{e}_{\varphi} \otimes \nabla_{s} v_{\varphi}
$$

and, since $v_{\varphi}$ depends only on $s, \nabla_{s} v_{\varphi} \cdot \mathbf{e}_{\varphi}=0$. Moreover, by (4.8), $\nabla_{s} \mathbf{e}_{\varphi} \cdot \nabla_{s} \boldsymbol{\nu}=0$ and so we conclude that

$$
\operatorname{div}_{s} \mathbf{a}=\sigma_{2} v_{\varphi} \operatorname{div}_{s} \mathbf{e}_{\varphi}+\mathbf{e}_{\varphi} \cdot \nabla_{s}\left(\sigma_{2} v_{\varphi}\right) .
$$

By use of (4.8), and observing that both $v_{\varphi}$ and $\sigma_{2}$ are independent of $\varphi$, we see that $\operatorname{div}_{s} \mathbf{a}$ vanishes as well. This justifies neglecting the azimuthal component of the velocity field.

\section{Conclusions}

In this paper I illustrated a new kind of interface propagation arising in a biological context. I outlined the main steps that led to the evolution equation and explored by example some qualitative properties of its solutions. By studying the relaxation of a curve close to a circle and subject to motion by curvature, I showed that this decay law differs substantially from that of a twodimensional vesicle. Finally, the asymptotic relaxation of an axisymmetric vesicle to a sphere was studied analytically: it revealed some unexpected features of this motion, which might serve to guide further studies, possibly numerical ones.

\section{Acknowledgement}

It is a pleasure to acknowledge Professor Epifanio G. Virga, for his encouragement in pursuing this work and for useful comments on it.

\section{REFERENCES}

1. CAi, W. \& Lubensky, T. C. Covariant hydrodynamics of fluid membranes. Phys. Rev. Lett. 73, (1994) $1186-1189$.

2. CANHAM, P. B. The minimum energy of bending as a possible explanation of the biconcave shape of the human red blood cell. J. Theor. Biol. 26, (1970) 61-81.

3. Chopp, D. L. \& Sethian, J. A. Motion by intrinsic Laplacian of curvature. Interfaces and Free Boundaries 1, (1999) 107-123.

4. Coleman, B. D., Falk, R. S., \& Moakher, M. Space-time finite element methods for surface diffusion with applications to the theory of the stability of cylinders. SIAM J. Sci. Comput. 17, (1996) 1434-1448.

5. Deuling, H. J. \& Helfrich, W. The curvature elasticity of fluid membranes: a catalogue of vesicle shapes. J. Physique 37, (1976) 1335-1345.

6. Do CARMo, M. P. Differential Geometry of Curves and Surfaces. Prentice-Hall, Englewood Cliffs NJ, (1976).

7. DoI, M. \& Edwards, S. The Theory of Polymer Dynamics. Clarendon, Oxford (1986).

8. Dörries, G. \& Foltin, G. Energy dissipation of fluid membranes. Phys. Rev. E 53, (1996) 2547-2550.

9. Foltin, G. Dynamics of incompressible fluid membranes. Phys. Rev. E 49, (1994) 5243-5248.

10. GAGE, M. E. Curve shortening makes convex curves circular. Invent. Math. 76, (1984) 357-364.

11. Gage, M. \& Hamilton, R. S. The heat equation shrinking convex plane curves. J. Diff. Geom. 23, (1986) 69-96. 
12. Grayson, M. A. The heat equation shrinks embedded plane curves to round points. J. Diff. Geom. 26, (1987) 285-314.

13. IsRAelaChVILI, J. Intermolecular and Surface Forces, 2nd edn. Academic, San Diego CA, (1991).

14. JARić, M., SEIfERT, U., Wintz, W., \& Wortis, M. Vesicular instabilities: the prolate-oblate transition and other shape instabilities of fluid bilayer membranes. Phys. Rev. E 52, (1995) 6623-6633.

15. Jost, J. Riemannian Geometry and Geometric Analysis, 2nd edn. Springer, Heidelberg (1998).

16. Milner, S. T. \& SAfran, S. A. Dynamical fluctuations of droplet microemulsions and vesicles. Phys. Rev. A 36, (1987) 4371-4379.

17. OU-YANG, Z.-C. Anchor ring-vesicle membranes. Phys. Rev. A 41, (1990) 4517-4520.

18. OU-YANG, Z.-C. Selection of toroidal shapes of partially polimerized membranes. Phys. Rev. A 47, (1993) 747-749.

19. OU-YANG, Z.-C. \& HelfRICH, W. Bending energy of vesicle membranes: general expressions for the first, second and third variation of the shape energy and applications to spheres and cylinders. Phys. Rev. A 39, (1989) 5280-5288.

20. Petrov, A. G. The Lyotropic State of Matter. Molecular Physics and Living Matter Physics. Gordon and Breach, Amsterdam (1999).

21. Prudnikov, A. P., BryčKov, Yu. A., \& MaričEv, O. I. Integrals and Series, English edition. Gordon and Breach, Amsterdam (1990).

22. Rosso, R., Sonnet, A. M., \& Virga, E. G. Evolution of vesicles subject to adhesion. Proc. R. Soc. A 456, (2000) 1523-1545.

23. Rosso, R. \& Virga, E. G. Adhesive borders of lipid membranes. Proc. R. Soc. A 455, (1999) 41454168.

24. Schneider, M. B., Jenkins, J. T., \& WebB, W. W. Thermal fluctuations of large quasi-spherical bimolecular phospholipid vesicles. J. Physique 45, (1984) 1457-1472.

25. Seifert, U. Configurations of fluid membranes and vesicles. Adv. Phys. 46, (1997) 13-137.

26. SEIfERT, U. Membranes in flow fields: formalism and an application to fluctuating quasi-spherical vesicles in shear flow. Eur. Phys. J. B 8, (1999) 405-415.

27. Seifert, U., BERndL, K., \& Lipowsky, R. Shape transformations of vesicles: phase diagrams for spontaneous curvature and bilayer-coupling models. Phys. Rev. A 44, (1991) 1182-1202.

28. VIRGA, E. G. Variational Theories for Liquid Crystals. Chapman and Hall, London (1994). 